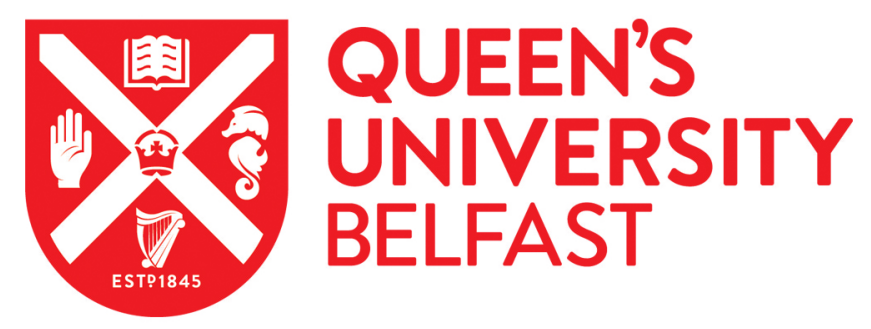

\title{
Reduction of threading dislocation density in top-down fabricated GaN nanocolumns via their lateral overgrowth by MOCVD
}

Zubialevich, V., McLaren, M., Pampili, P., Shen, J., Arredondo-Arechavala, M., \& Parbrook, P. J. (2020).

Reduction of threading dislocation density in top-down fabricated GaN nanocolumns via their lateral overgrowth by MOCVD. Journal of Applied Physics, 127(2), [025306]. https://doi.org/10.1063/1.5110602

Published in:

Journal of Applied Physics

Document Version:

Peer reviewed version

Queen's University Belfast - Research Portal:

Link to publication record in Queen's University Belfast Research Portal

Publisher rights

(c) 2019 The Authors. This work is made available online in accordance with the publisher's policies. Please refer to any applicable terms of use of the publisher.

\section{General rights}

Copyright for the publications made accessible via the Queen's University Belfast Research Portal is retained by the author(s) and / or other copyright owners and it is a condition of accessing these publications that users recognise and abide by the legal requirements associated with these rights.

Take down policy

The Research Portal is Queen's institutional repository that provides access to Queen's research output. Every effort has been made to ensure that content in the Research Portal does not infringe any person's rights, or applicable UK laws. If you discover content in the Research Portal that you believe breaches copyright or violates any law, please contact openaccess@qub.ac.uk. 


\title{
Reduction of threading dislocation density in top-down fabricated GaN nanocolumns via their lateral overgrowth by MOCVD
}

\author{
Vitaly Z. Zubialevich ${ }^{a}$, Mathew McLaren ${ }^{b}$, Pietro Pampili ${ }^{a}$, John Shen ${ }^{c}$, \\ Miryam Arredondo-Arechavala ${ }^{b,}{ }^{*}$, Peter J. Parbrook ${ }^{a, d}, \dagger$
}

${ }^{a}$ Tyndall National Institute, University College Cork, Cork, T12 R5CP, Ireland.

${ }^{b}$ Centre for Nanostructured Media, School of Mathematics and Physics, Queen's University, Belfast, BT7 1NN, N. Ireland;

${ }^{*}$ E-mail: m.arredondo@qub.ac.uk

c Electrical and Computer Engineering Department, Illinois Institute of Technology, Chicago, IL 60616, Illinois, USA

${ }^{d}$ School of Engineering, University College Cork, Cork, Ireland; †E-mail: p.parbrook@ucc.ie

\section{Abstract}

Reduction of threading dislocation density in top-down fabricated GaN nanocolumns (NCs) via their successive lateral shrinkage by anisotropic wet etch and lateral overgrowth by MOCVD is studied by transmission electron microscopy (TEM). The fabrication process involves a combination of dry and wet etches to produce NC arrays of low fill factor $(<5 \%)$ which are then annealed and laterally overgrown to increase the array fill factor to around $20-30 \%$. The resulting NC arrays show a reduction in treading dislocation density of at least 25 times, allowing for the reduction in material volume due to the array fill factor, with dislocations being observed to bend into the voids between NCs during the overgrowth process.

\section{Introduction}

Gallium nitride and other wide bandgap semiconductors have received much attention in recent years for a variety of applications in optoelectronics and high temperature, high power devices [1-4]. Film growth on substrates such as silicon and sapphire is the most common device preparation method $[5,6]$, with the aim of producing transistors with high electron mobility. However, a key issue with the growth of these films is the presence of threading dislocations (TDs), a concern when fabricating GaN-based semiconductor devices due to the negative effects they may have on device quality such as increased current leakage [7, 8], decreased efficiency and lifetime [9], disrupted electric field distribution and a premature breakdown due to microplasmas $[10,11]$. Dislocation densities in the range of $10^{8} \mathrm{~cm}^{-2}$ to $10^{10} \mathrm{~cm}^{-2}$ are often recorded for few-to-several micrometer-thick heteroepitaxially grown GaN films $[12,13]$. While light emitting diodes, which only need to operate in forward bias, may be fabricated from material with such high TD densities (TDDs) [10,14], for high 
power or high temperature applications it is imperative that TDD should be considerably reduced to produce quality devices.

One such method of TDD reduction, for example, is the annealing of nanostructured GaN to temperatures of $900^{\circ} \mathrm{C}$ [15]. Nanostructuring of GaN is a proven method for TD reduction. This can include the extra growth steps such as the creation of interlayers of $\operatorname{SiN}_{x}$ or $\operatorname{ScN}$ which act to stop dislocations from propagating $[16,17]$. Another approach for dislocation reduction involves epitaxial lateral overgrowth [18, 19], which is a second stage of GaN growth after the initial GaN formation and patterning (typically on the microscale). The latter has been very successful for reducing TDDs by several orders of magnitude, closer to values of $10^{6}-10^{7} \mathrm{~cm}^{-2}$. Finally, nanostructured GaN in the form of nanocolumn (NC) arrays is found to be an effective method of dislocation reduction [20]. Due to the higher surface-to-volume area it is potentially easier to achieve dislocation reduction in nanostructured materials. Notably, when prepared by a bottom-up approach, GaN NCs with a small enough diameter are observed to be dislocation free due to dislocation bending towards NC sidewalls at early stages of growth [21]. In general this method is less suitable for mass-production of potential devices due to (a) unavoidable variability in individual NCs dimensions, particularly, height, (b) apex tops of grown NCs (when grown Ga-polar), (c) limited achievable fill factor of their arrays, and finally (d) issues with controlling doping levels. In contrast, many of these issues may be avoided by fabricating NC arrays using "top-down" fabrication approaches [22].

Here we discuss effects on TDD within GaN NC arrays caused by the implementation of a hybrid top-down-regrowth fabrication approach: using a dry/wet etching process on a grown by metalorganic chemical vapor deposition (MOCVD) film to produce NC arrays followed by its lateral annealing/overgrowth. The main result is that majority of TDs successfully bend away from the NC region into the gaps inbetween ultimately significantly reducing the TDD in the array.

\section{Experiment}

Initial GaN/sapphire templates were prepared by MOCVD in a showerhead type 3x2" AIXTRON reactor using trimethylgallium and ammonia as $\mathrm{Ga}$ and $\mathrm{N}$ precursors, disilane as a silicon dopant source and hydrogen as the carrier gas. After a short AlN connecting layer on AlN/sapphire wafers (Kyma Technologies), a straightforward GaN growth at $1060^{\circ} \mathrm{C}, 150 \mathrm{mbar}$ and V/III ratio of 1000 was undertaken where no disilane was supplied for the first $200 \mathrm{~nm}$ i.e. material was nominally undoped ( $u$-GaN). This was followed by $1.8 \mu \mathrm{m}$ of $n^{+}-\mathrm{GaN}$ with a nominal [Si] $\sim 5 \times 10^{18} \mathrm{~cm}^{-3}$, and finally an $n^{-}$GaN drift region was $3 \mu \mathrm{m}$ with [Si] $1 \times 10^{17} \mathrm{~cm}^{-3}$. The growth was monitored optically using a LayTec EpiCurve ${ }^{\circledR}$ TT tool containing reflectometer, pyrometer and wafer bowing detection system. 
A colloid monolayer of silica nanospheres (SNSs) self-assembled on the water surface in the form of locally ordered (in a hexagonal lattice) closely packed array was transferred onto GaN wafer using a scooping approach $[23,24]$. The SNS diameter was $750 \pm 40 \mathrm{~nm}$. This SNS monolayer was used as a hard mask for dry inductively coupled plasma (ICP) etching to form GaN NCs. A KOH-based (using AZ 400K developer) wet etch was then applied to improve the as dry etched NC sidewall surface morphology (requiring an etch time of around 4-8 hours at room temperature) and laterally shrink the columns to a final diameter of around $150 \mathrm{~nm}$ (necessitating an additional 12-20 hours etching). This step allows removal of any surface damage introduced by the dry etch as well as a significant fraction of material volume with its built-in dislocations. The exact fabrication procedure has recently been briefly reported in Ref. [25], and further details will be published elsewhere [26]. The arrays of GaN NCs were finally brought back to the reactor for annealing and regrowth at $950^{\circ} \mathrm{C}$ in nitrogen to form six vertical $m$-plane sidewall facets and laterally expand the NCs. The optimization strategy for the regrowth conditions and details of NC array regrowth process will be presented separately [27].

The epitaxial structures, and NC arrays fabricated from them, were characterized by X-ray diffraction (XRD) using a PANalytical X'pert PRO XRD system. Symmetric 0004 $2 \Theta-\omega$ scans and the corresponding symmetric 0004 reciprocal space maps were measured to determine the strain status of the prepared samples.

Prior to transmission electron microscopy (TEM) analysis, studied samples were prescreened using Thermo Scientific ${ }^{\mathrm{TM}}$ Quanta $^{\mathrm{TM}} 650$ and JEOL JSM6500F scanning electron microscopes (SEM). The selected samples were prepared into cross-sections for TEM characterization using conventional mechanical dimpling polishing process followed by argon ion polishing at $3.5 \mathrm{kV}$ and a final low energy polish at $0.5 \mathrm{kV}$ to final thickness, $t_{f} \approx 200 \mathrm{~nm}$. Samples were characterized using a FEI Tecnai F20 at $200 \mathrm{kV}$ and a FEI CM20 at $200 \mathrm{kV}$.

To determine TDD we apply a direct approach from TEM images, based on the method of Martin et al. [28] which is commonly used. This method relies on specimen thickness, $t_{f}$ and number of dislocations crossing an arbitrary line across the sample image, in our case perpendicular to the growth direction.

There are other methods to determine TDD in epitaxial films, as reviewed by Pandey et al. [29], such as from XRD or using the density of pits induced by chemical etching (EPD). However, both of these are ill-suited for application to nanocolumn arrays. For XRD the rocking curve width typically used is influenced by the mutual tilts of the NCs rendering the approach invalid. EPD, which tends to underestimate TDD at higher densities in any case, cannot be applied as $\mathrm{KOH}$ based etchants normally used are those that are used to modify NC diameter and shape, and would destroy our NCs completely using conditions necessary to induce pit on a c-plane surface.

The array fill factor was calculated as the ratio between area of an average NC cross section (circular for as wet etched NCs and hexagonal for overgrown ones) and area 
of NC array cell. The latter in our case is the area of hexagon circumscribed to a circle of diameter equal to array pitch (or the average diameter of SNSs used to produce the array). The necessary values for the calculations were determined from SEM images of the corresponding NC arrays.

\section{Results and Analysis}

Figure 1 shows a high angle annular dark field (HAADF) image demonstrating a typical overview of a GaN film grown onto a sapphire substrate (with an AlN seed layer) using MOVPE. The target film thickness was $5 \mu \mathrm{m}$ with a measured thickness of $4.95 \mu \mathrm{m}$. The interfaces between $u^{-}, n^{+-}$and $n^{-}$-doped GaN are highlighted by the dotted lines. The interfaces cannot be determined quantitatively due to the extremely subtle content of silicon doping concentrations. However, the aforementioned measurement of the total film thickness shows that the GaN is growing as expected with minor variation suggesting that the position of the interfaces is approximately correct.

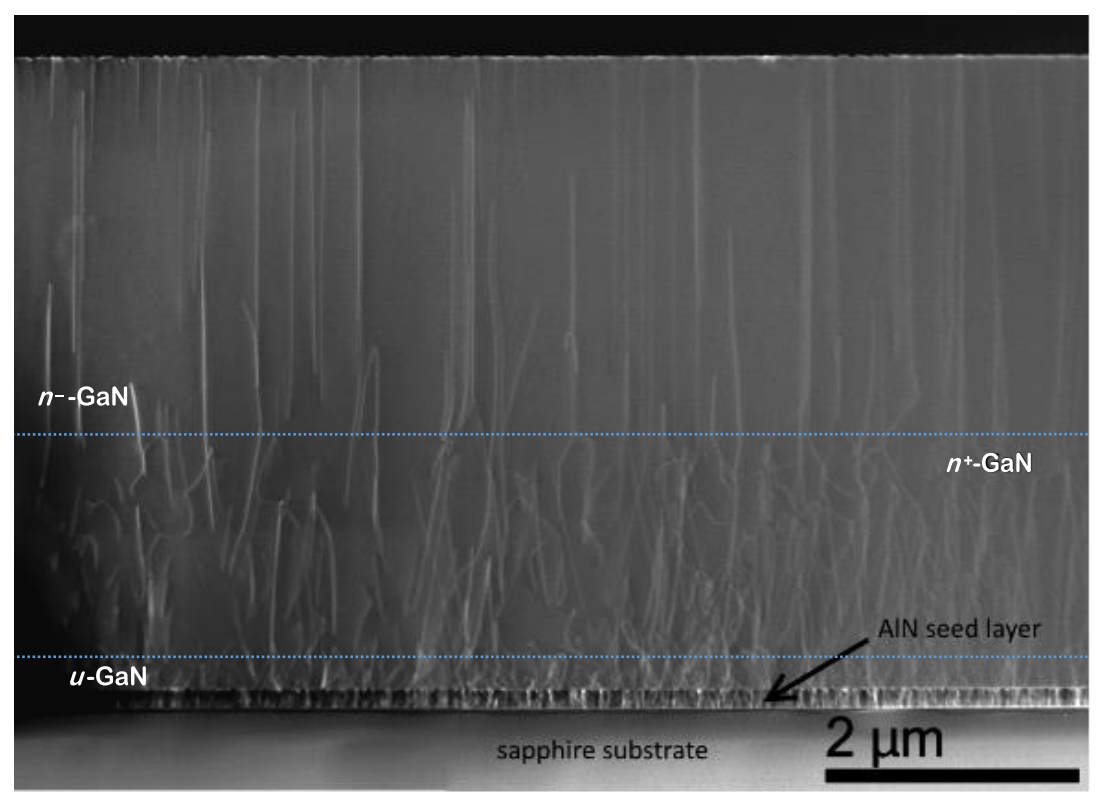

Fig. 1. - HAADF-STEM image of a typical GaN film prior to etching. Dotted lines indicate the expected interfaces between the $u-, n^{+}$-and $n^{-}$-doped regions.

The position of the second interface (between $n^{+}$- and $n^{-}$-GaN) roughly correlates with a change in the TD's behavior, as clearly observed in the top layer, labeled $n^{-}-\mathrm{GaN}$. In contrast to the TDs in the $n^{+}-\mathrm{GaN}$, those in $n^{-}$GaN region appear to remain straight until they terminate at the top of the film. This fits with the reports of Dadgar et al. [30] where dislocations in Si-doped GaN tend to bend due to climb before becoming more vertical in an undoped GaN overlayer. Such dislocation inclination with $\mathrm{Si}$ doping has been observed by other authors including, for example, Forghani et al. [31] who explained it by Ga vacancy assisted dislocation "climb". Due to Si doping, Ga vacancy energy formation decreases with increase of Fermi level [32], which 
increases Ga vacancy concentration in $n$-GaN generally, while locally their formation is promoted by preferential incorporation of $\mathrm{Si}$ adatoms at the compressive side of the strain dipole at edge/mixed type dislocation core [31].

As is seen in Figure 1, the dislocation density decreases with layer thickness, as expected. There is a strong decrease in TDD with thickness within the first $\sim 2 \mu \mathrm{m}$ from the substrate, which then stabilizes with a small decrease beyond this point. At the film top, a TDD of $\sim 2 \times 10^{9} \mathrm{~cm}^{-2}$ was determined which follows closely to literature values of $10^{8}-10^{10} \mathrm{~cm}^{-2}$ for similar films.

A general overview of the GaN NC arrays is presented in Fig. 2. Figures 2, $a$ and 2, $b$ show the consistency of the $\sim 1.6 \mu \mathrm{m}$ tall nanocolumns pre- and post-overgrowth, respectively. There is some variation in lateral NC size which may result from a variety of effects including variations in initial nanosphere diameter, inhomogeneities of the dry ICP and wet $\mathrm{KOH}$-based etches prior to the overgrowth stage (Fig. 2, a) as well as local growth rate effects due to any array inhomogeneities and initial NC dimensions. There is some variation in the etch depth of the NCs, of the order of 100 $\mathrm{nm}$, which relates primarily to deeper etching around defects in the $\mathrm{NC}$ hexagonal array. The NC tops are primarily defined by the initial layer growth, which has a typical root-mean-square roughness, from atomic force microscopy, of less than $1 \mathrm{~nm}$ [33]. The final NC top height variation is not expected to substantially increase as the wet etch is highly anisotropic (NCs shrink laterally but no $c$-plane etch happens [34]) and the regrowth conditions $\left(950^{\circ} \mathrm{C}, \mathrm{N}_{2}\right.$ ambient, high $\mathrm{V} / \mathrm{III}$ ratio) are chosen to minimize $c$-plane growth. Full details on the regrowth procedure will be published elsewhere [27]; however, the key feature is that the slow c-plane growth is determined by the fact that if no TMGa is supplied at the applied conditions, there is a strong decomposition of GaN from $c$-plane facets of GaN NCs [15]. Supplying of TMGa during the overgrowth step here simply slows down the decomposition, so that zero, or even a slightly negative, growth rate is expected for NC $c$-plane facets.

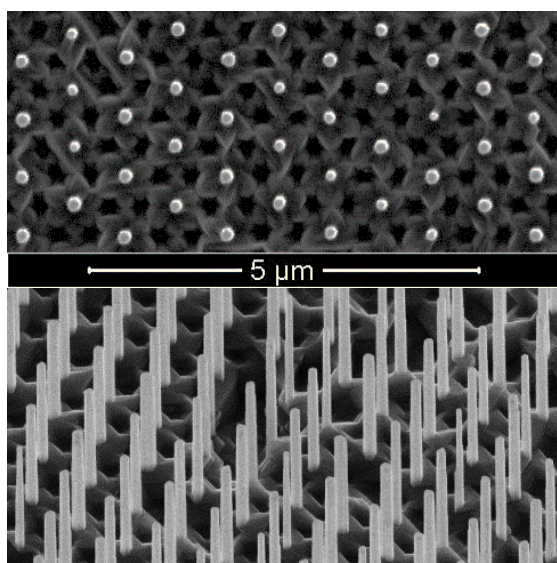

(a)

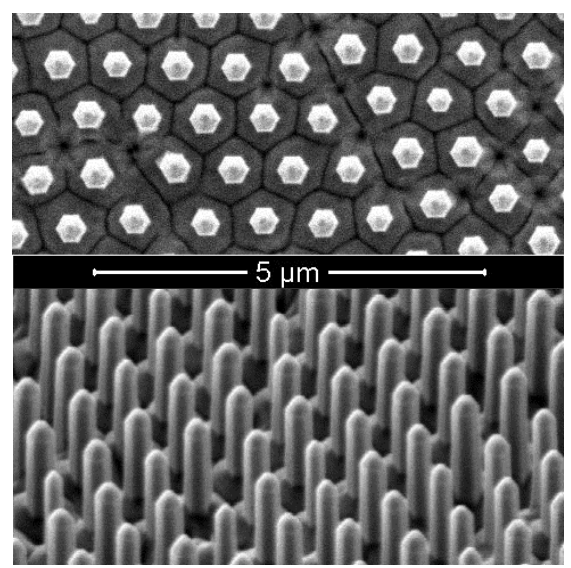

(b)

Fig. 2. - SEM micrographs of GaN NC (a) after $\sim 20$ hour long KOH-based wet etching and $(b)$ after an overgrowth at $950^{\circ} \mathrm{C}$ in nitrogen ambient (upper parts: top view; bottom parts: $45^{\circ}$ tilted view). 
To investigate the strain state of the different layers of initial GaN templates and GaN NCs, a separate structure was grown, processed and characterized by XRD. The only difference from the structure discussed above was an increase in the initial $u$-GaN thickness from $200 \mathrm{~nm}$ to $500 \mathrm{~nm}$, accompanied by an equivalent decrease in the thickness of $n^{+}-\mathrm{GaN}(1500 \mathrm{~nm}$ vs. $1800 \mathrm{~nm}$ ). In Fig. 3, $a$, a typical symmetric (0004) $2 \theta-\omega$ XRD reflection curve of the $u-\mathrm{GaN} / n^{+}-\mathrm{GaN} / n^{-}-\mathrm{GaN}$ initial template (curve 1 ) is compared to that for the same sample after $\mathrm{NC}$ array formation (curve 2 ).

For the initial template, two peaks are visible, both corresponding to compressively strained GaN. The lower intensity peak at $2 \theta=72.82^{\circ}$ can be attributed to the undoped $u$-GaN grown directly on the AlN nucleation layer. At the growth temperature, this layer is expected to be under slight compressive strain due to the larger $a$ lattice parameter of GaN $(3.189 \mathrm{~nm})$, compared to AlN $(3.111 \mathrm{~nm})[35,36]$. Due to the larger thermal expansion coefficient of sapphire $\left(7.5 \cdot 10^{-6} \mathrm{~K}^{-1}\right)$ as compared to $\mathrm{GaN}\left(5.6 \cdot 10^{-6} \mathrm{~K}^{-1}\right)$ [37], the $u$-GaN layer experiences strong compressive strain upon cooling to room temperature.

The dominant peak at $2 \theta=72.89^{\circ}$ corresponds to the doped $n^{+}$and $n^{-}$GaN layers. These layers are under tensile strain at the growth temperature due to doping with silicon [30, 31, 38] but become slightly compressive on cooldown due to the GaN/sapphire thermal expansion coefficient difference. Although with reduction of

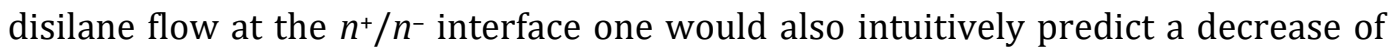
tensile strain in $n^{-}$-GaN compared to $n^{+}-\mathrm{GaN}$, we do not see splitting of this peak into two separate ones. Furthermore, no such strain decrease was visible from the in-situ curvature measurement as the $n^{-}$-GaN layer was grown.

Upon NC formation (curve 2 in Fig. 3,a), a third peak appears that matches perfectly with the theoretical value for unstrained GaN. It should be noted that to be able to see some XRD signal from the nanopatterned material, these data have been obtained for a sample that was only dry etched (hence the NC array fill factor was significantly larger than that for the array presented in Fig. 2, $a$ for example). Through removal of material to form a NC with ICP etching, the pre-existing strain within the NC body is free to relax, and therefore it is quite reasonable to assign this peak to GaN NCs.

Additionally, the latter is strongly supported from the comparison of 0004 reciprocal space maps measured before and after formation of $\mathrm{NC}$ array (Fig. 3, $b$ and 3, respectively), one can see that the new peak is visibly broader in the horizontal coordinate corresponding in this case to pure $\omega$ angle. By appropriately analyzing selected linescans from the reciprocal space maps, the rocking curve half-widths for the dominant peak and additional shoulder have been estimated to be $\sim 80$ and $\sim 140$ arcsec, respectively. We attribute the broadening of the relaxed GaN peak to small mutual tilts of the NCs induced by random distribution of the pre-existing dislocations in and between the NCs, as will be shown in the TEM data. 
While generation of dislocations at the edges of extra half-planes in materials grown on non-lattice-matched substrates is an energetically costly process (there is a line energy associated with a dislocation [39]), it nevertheless occurs as the formation of the extra half-planes reduces biaxial stress in the structure and thus system total energy. Upon formation of NCs from dislocated bulk GaN, however, biaxial strain is free to relax (as seen from the XRD in Fig. 3), and thus the energy associated with those dislocations still in the NC bodies becomes a non-compensated/excess energy. The system should be willing to get rid of this energy by reducing the dislocation lengths. Annealing during the overgrowth step of our fabrication technique can provide suitable conditions for this process.
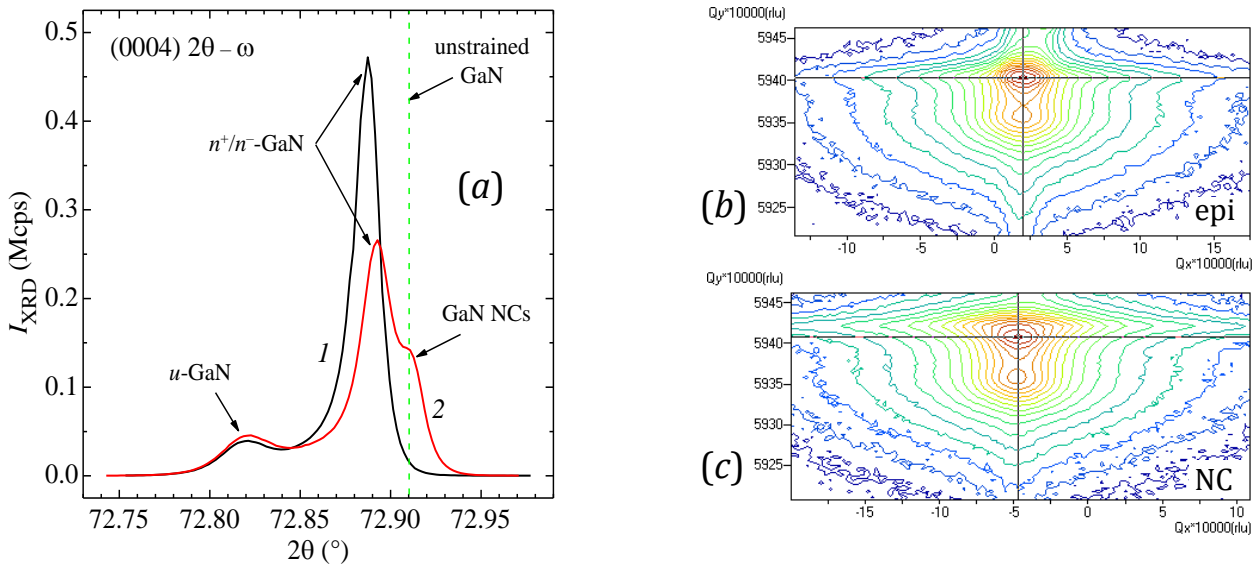

Fig. 3. - (0004) XRD 2 $\theta-\omega$ scans of a GaN before (curve 1) and after (curve 2) NC array formation $(a)$ and the corresponding reciprocal space maps $(b, c)$.

The above statement is corroborated by the absence of observable TDs within bodies of these 'extremely' shrunk and then overgrown NCs (see Fig. 4 for a typical example). Instead of propagating into the NC bodies, TDs tend to bend in the gaps in between them; as highlighted by arrows in Fig. 4. Although it should be mentioned that the the number of examined NCs does not allow a statistically meaningful quantitative estimation regarding the effective TDD for this sample, we conclude that most dislocations have been removed together with material during the dry etch and subsequent 'extreme' NC shrinkage (as shown in Fig. 2,a). Any residual dislocations are then bent out of the NCs by the regrowth step, which was carried out at an elevated temperature $\left(950^{\circ} \mathrm{C}\right)$.

To be able to do a more quantitative analysis, another array of NCs was fabricated where the lateral NC shrinkage prior to overgrowth was somewhat reduced, giving seed NC diameters of $\sim 200 \mathrm{~nm}$, as opposed to $50-150 \mathrm{~nm}$ in the previous case. TEM analysis on TDs was carried out using the invisibility criterion $(g \cdot b=0)$ with reflective plane vectors $g=\langle 11 \overline{2} 0\rangle$ and $g=\langle 0002\rangle$. Analysis of Burgers vectors $b$ shows the majority of dislocations are edge or mixed type, and as expected only a few pure 
screw-type dislocations were identified. This is consistent with sample data based on XRD rocking curves.

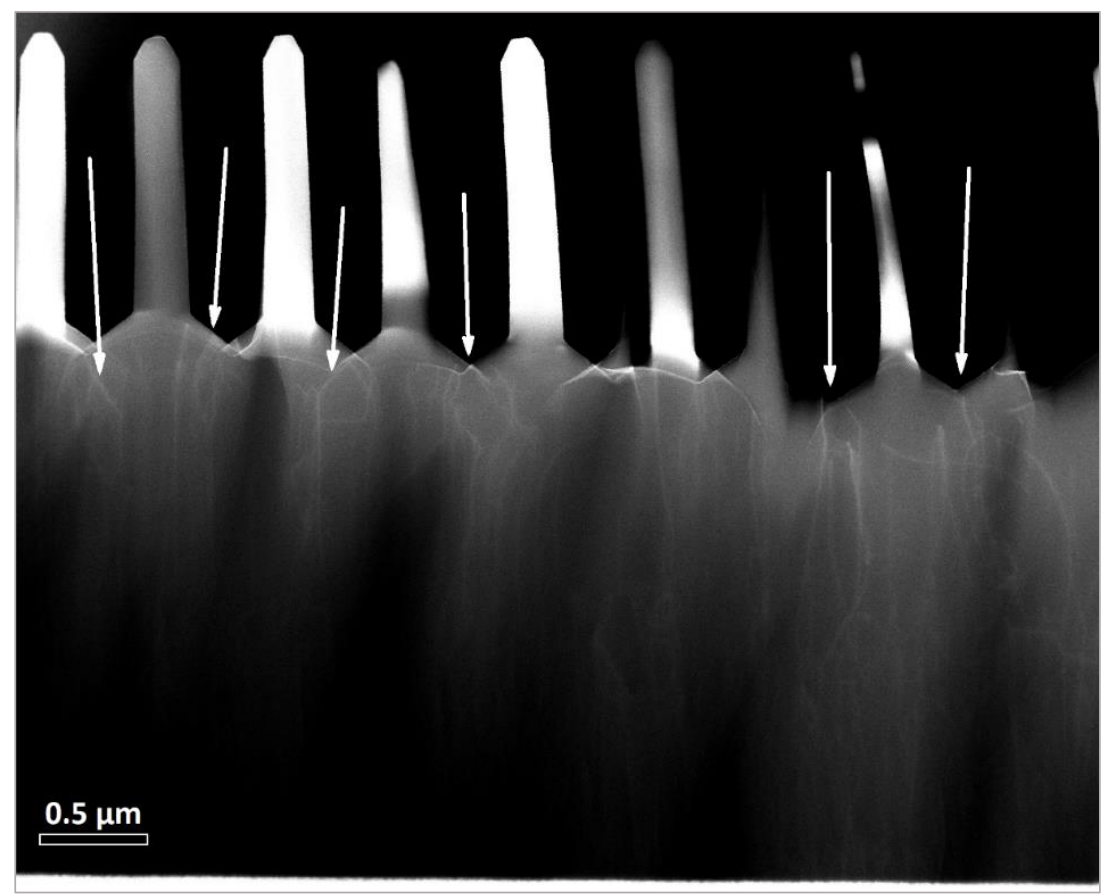

Fig. 4. - HAADF-STEM image of the GaN NC array upon 'extreme' wet etch and lateral overgrowth. Note the absence of dislocations propagating into the NCs at their base.

Measurements of the dislocation density in the overgrown NCs find a value of $\sim 2.4 \times 10^{7} \mathrm{~cm}^{-2}$ which, taking into account resulting fill-factor of $\sim 30 \%$ corresponds to effective TDD of $\sim 8 \times 10^{7} \mathrm{~cm}^{-2}$. This is both a significant reduction compared to the aforementioned value of $2 \times 10^{9} \mathrm{~cm}^{-2}$ for an unetched film and the typical reported literature values. The observed TDD reduction by a factor of about 25 is considerably higher than the value simply estimated from amount of material removed by formation and lateral shrinkage of NCs. The latter figure is the ratio of areas of hexagonal footprint of a NC and its section after wet etch shrinkage. Taking into account array pitch defined by the initial diameter of SNSs used $(\approx 750 \mathrm{~nm})$ and the average diameter of GaN NC after their lateral shrinkage by wet etch $(\approx 200 \mathrm{~nm})$, the proportion of original material in our NCs is estimated to be about $6.5 \%$ of total. This accounts for a factor of about 15.5 improvement in TDD and thus the rest can be attributed to dislocation bending out of NC bodies into the gaps between the NCs as shown below.

Various examples of the bending of TDs around the depth corresponding to the NC bases are shown more detailed in Fig. 5 . Unlike in the 'extremely' wet etched sample of Fig. 4, some rare TDs are seen to propagate through to the end of the NC (Fig. 5, $a$ and 5,e). As already demonstrated in Fig. 1, many TDs will travel to the top of the GaN film. While the etching stage of NC fabrication will remove a large amount of material containing TDs; thus, it is expected that some of them will remain within the NCs. Due 
to the high energy associated with dislocations [40] and vicinity of voids in between NCs, one could expect that if a dislocation can move it can also bend out of a NC to reduce the overall system energy. We, however, consider such bending to be possible only for TDs terminating at NC sidewalls upon their lateral shrinkage. This is further supported by the absence of dislocations terminating on the sidewalls of all examined NCs (over $\sim 80 \mathrm{NCs}$ characterized). We believe that all such TDs actually have either bent out during the wet etch or during the annealing/overgrowth stage. From this point of view, it is also clear to understand why TDs terminating at the top $c$-plane facets of NCs do not seem to bend. First of all, to finally shorten their length by bending out and migrating with its termination point down to the NC foot, such dislocations have to increase their length first to reach $c$-plane $/ m$-plane intersection, for which, the thermal energy at regrowth conditions may not be sufficient. Secondly termination points of TDs are often decorated with V-pits [41] and thus can be strongly pinned to these surface defects.

Aside from the TDs within the NC bodies, it appears that many TDs bend near the top of the 'layer' region just below the start of the NC region. This is clear in Fig. 5, $b$ where four TDs, two of which appear to be propagating directly into the NCs, bend into the same region in the middle, in-between the NCs. Analysis of these TDs shows that such bending occurs in a region 3.0-3.2 $\mu \mathrm{m}$ above the base of the GaN layer which corresponds to a region $\sim 300 \mathrm{~nm}$ below the bottom of the troughs between the NCs.

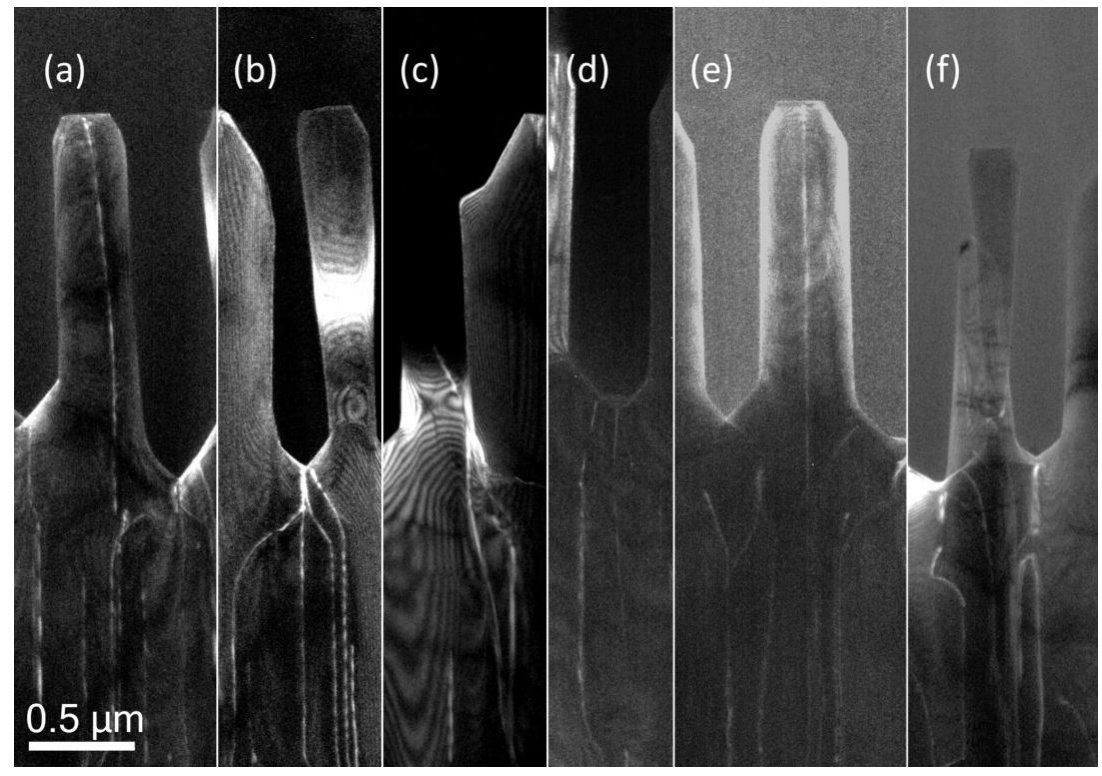

Fig. 5. - Dark field TEM micrographs of laterally overgrown GaN, demonstrating the bending of the TDs away from the NCs in the nominally overgrown regions.

The observed dislocation bending differs from that in bottom-up fabricated (grown) GaN NCs. While completely dislocation-free NCs are claimed only in the case of quite small diameters (typically $\lesssim 100 \mathrm{~nm}$ ), dislocation filtering is also happening for larger NCs. However, in this case, dislocation bending happens already within the NC body, 
typically in its lower part $[21,42]$ with the propagation height of the dislocations increasing with NC diameter [21]. Generally, the bending of dislocations is welldocumented in GaN $[13,21]$, particularly as a result of post-growth thermal annealing [15] or lateral epitaxial overgrowth [13] where so called image forces are considered to be the main mechanism for such dislocation movements [43]. From comparison of the two NC samples here, one can conclude that a dislocation in a GaN NC needs to be closer than at least 50-70 nm from the surface for image forces to be able to push it away from NC body. Our estimation matches pretty well with theoretical figure of about 200 Burgers vectors ( $\sim 62 \mathrm{~nm}$ for edge dislocations in GaN) from the free surface in a nanocrystal where image forces become essentially non-zero [44]. This perhaps can explain why no TDs could be observed in our 'extremely' wet etched and overgrown NCs, but a small number of them managed to remain in the sample that was not so strongly shrunk before overgrowth. This actually might be also the reason why dislocations simply do not form in bottom-up grown GaN NCs of small enough diameters [21].

From the TEM analysis it can be then inferred that the rough surface in the areas inbetween NCs (Fig. 2,a) upon annealing/growth stage converts into thermodynamically stable semipolar $\{1 \overline{1} 01\}$ facets. These form pedestals at the base of each NC and valleys, with the deepest regions of no material where adjacent pedestals intersect. These valleys act as attractors for dislocations (bent out from NC bodies and those etched away from them) as they minimize TD lengths, compared to those in the underlying GaN films, and thus the overall system energy. That is the main reason why most of our NCs are free of dislocations all the way to the NC base.

\section{Conclusions}

By using a novel hybrid top-down-regrowth method combining MOVPE, ICP etching with silica nanosphere monolayer as a hard mask, lateral shrinkage with a $\mathrm{KOH}$-based wet etch and a further lateral overgrowth step, gallium nitride nanocolumns can be fabricated with a very low dislocation density relative to other current top-down methods. Transmission electron microscope dark field imaging and analysis of the threading dislocations shows a directional change as they approach the NCs, bending into the gaps between them. The analysis confirms that this novel fabrication method allows for a significant reduction in dislocation density compared to as-grown GaN films. By implementing an 'extreme' lateral shrinkage of NCs (down to diameters of about 50-150 $\mathrm{nm}$, i.e. just before some initially thinnest NCs may start to disappear) before overgrowth, it was possible to eliminate dislocations practically completely. In the other studied case on not so 'extremely' shrunk NCs, TDD values of about $2 \times 10^{9} \mathrm{~cm}^{-2}$ in bulk layer as compared to $2.4 \times 10^{7} \mathrm{~cm}^{-2}\left(8 \times 10^{7} \mathrm{~cm}^{-2}\right.$ taking into account array fill factor) in the overgrown GaN NC arrays have been estimated, giving improvement factor of about 25. Those NCs with remained dislocations are nevertheless characterized by sidewall surfaces free of them (the only dislocations remaining are those completely "buried" in the middle of NC bodies). Taking into 
account this feature, one can predict that besides using these NCs for applications of power electronics (the intended use of the present NC arrays was Schottky diodes [45]), they might be especially promising for optoelectronic devices based on heterostructures with a core-shell architecture.

\section{Acknowledgements}

This study was carried out with financial support from Irish Higher Education Authority Programme for Research in Third Level Institutions Cycles 4 and 5 via the INSPIRE and TYFFANI projects, Science Foundation Ireland (Grants no. SFI13/US/I2860, SFI-18/TIDA/6066), from the Department for the Economy Northern Ireland under the US-Ireland R\&D Partnership Programme (Grant no. USI-058), and from U.S. National Science Foundation under Grant EECS-1407540. One author (PJP) acknowledges funding from SFI Engineering Professorship scheme (SFI/07/EN/E001A). Many thanks to Dr. Martina Luysberg of the Ernst-Ruska Centre, Forschungszentrum Jülich for access to the CM20 TEM.

\section{References}

[1] E. Calleja, M.A. Sánchez-García, F.J. Sánchez, F. Calle, F.B. Naranjo, E. Muñoz, U. Jahn, K. Ploog, Luminescence properties and defects in GaN nanocolumns grown by molecular beam epitaxy, Physical Review B, 62 (2000) 16826-16834.

[2] R. Birkhahn, A.J. Steckl, Green emission from Er-doped GaN grown by molecular beam epitaxy on Si substrates, Applied Physics Letters, 73 (1998) 2143-2145.

[3] Y. Huang, X. Duan, Y. Cui, C.M. Lieber, Gallium Nitride Nanowire Nanodevices, Nano Letters, 2 (2002) 101-104.

[4] Z. Zhong, F. Qian, D. Wang, C.M. Lieber, Synthesis of p-Type Gallium Nitride Nanowires for Electronic and Photonic Nanodevices, Nano Letters, 3 (2003) 343-346. [5] T. Lei, M. Fanciulli, R.J. Molnar, T.D. Moustakas, R.J. Graham, J. Scanlon, Epitaxial growth of zinc blende and wurtzitic gallium nitride thin films on (001) silicon, Applied Physics Letters, 59 (1991) 944-946.

[6] D. Doppalapudi, E. Iliopoulos, S.N. Basu, T.D. Moustakas, Epitaxial growth of gallium nitride thin films on A-Plane sapphire by molecular beam epitaxy, Journal of Applied Physics, 85 (1999) 3582-3589.

[7] S.W. Lee, D.C. Oh, H. Goto, J.S. Ha, H.J. Lee, T. Hanada, M.W. Cho, T. Yao, S.K. Hong, H.Y. Lee, S.R. Cho, J.W. Choi, J.H. Choi, J.H. Jang, J.E. Shin, J.S. Lee, Origin of forward leakage current in GaN-based light-emitting devices, Applied Physics Letters, 89 (2006) 132117.

[8] M.F. Schubert, S. Chhajed, J.K. Kim, E.F. Schubert, D.D. Koleske, M.H. Crawford, S.R. Lee, A.J. Fischer, G. Thaler, M.A. Banas, Effect of dislocation density on efficiency droop in GaInNGaN light-emitting diodes, Applied Physics Letters, 91 (2007) 231114.

[9] M. Ťapajna, S.W. Kaun, M.H. Wong, F. Gao, T. Palacios, U.K. Mishra, J.S. Speck, M. Kuball, Influence of threading dislocation density on early degradation in AlGaN/GaN high electron mobility transistors, Applied Physics Letters, 99 (2011) 223501.

[10] E. Cicek, Z. Vashaei, C. Bayram, R. McClintock, M. Razeghi, M.P. Ulmer, Comparison of ultraviolet APDs grown on free-standing GaN and sapphire substrates, SPIE, 2010. [11] C. Gupta, Y. Enatsu, G. Gupta, S. Keller, U.K. Mishra, High breakdown voltage p-n diodes on GaN on sapphire by MOCVD, physica status solidi (a), 213 (2016) 878-882. 
[12] D.S. Peng, Y.C. Feng, W.X. Wang, X.F. Liu, W. Shi, H.B. Niu, High-quality GaN films grown on surface treated sapphire substrate, Journal of Physics D: Applied Physics, 40 (2007) 1108-1112.

[13] S.E. Bennett, Dislocations and their reduction in GaN, Materials Science and Technology, 26 (2010) 1017-1028.

[14] S.D. Lester, F.A. Ponce, M.G. Craford, D.A. Steigerwald, High dislocation densities in high efficiency GaN-based light-emitting diodes, Applied Physics Letters, 66 (1995) 1249-1251.

[15] M. Conroy, H.N. Li, V.Z. Zubialevich, G. Kusch, M. Schmidt, T. Collins, C. Glynn, R.W. Martin, C. O'Dwyer, M.D. Morris, J.D. Holmes, P.J. Parbrook, Self-Healing Thermal Annealing: Surface Morphological Restructuring Control of GaN Nanorods, Crystal Growth \& Design, 16 (2016) 6769-6775.

[16] M.J. Kappers, M.A. Moram, Y. Zhang, M.E. Vickers, Z.H. Barber, C.J. Humphreys, Interlayer methods for reducing the dislocation density in gallium nitride, Physica B: Condensed Matter, 401-402 (2007) 296-301.

[17] M.A. Moram, Y. Zhang, M.J. Kappers, Z.H. Barber, C.J. Humphreys, Dislocation reduction in gallium nitride films using scandium nitride interlayers, Applied Physics Letters, 91 (2007) 152101.

[18] B.A. Haskell, F. Wu, M.D. Craven, S. Matsuda, P.T. Fini, T. Fujii, K. Fujito, S.P. DenBaars, J.S. Speck, S. Nakamura, Defect reduction in (1120) a-plane gallium nitride via lateral epitaxial overgrowth by hydride vapor-phase epitaxy, Applied Physics Letters, 83 (2003) 644-646.

[19] B.M. Imer, F. Wu, S.P. DenBaars, J.S. Speck, Improved quality (1120)a-plane GaN with sidewall lateral epitaxial overgrowth, Applied Physics Letters, 88 (2006) 061908.

[20] F. Qian, Y. Li, S. Gradečak, D. Wang, C.J. Barrelet, C.M. Lieber, Gallium NitrideBased Nanowire Radial Heterostructures for Nanophotonics, Nano Letters, 4 (2004) 1975-1979.

[21] K. Kishino, S. Ishizawa, Selective-area growth of GaN nanocolumns on $\mathrm{Si}(111)$ substrates for application to nanocolumn emitters with systematic analysis of dislocation filtering effect of nanocolumns, Nanotechnology, 26 (2015) 225602.

[22] P.R. Narangari, S.K. Karuturi, M. Lysevych, H.H. Tan, C. Jagadish, Improved photoelectrochemical performance of GaN nanopillar photoanodes, Nanotechnology, 28 (2017) 154001.

[23] M. Conroy, V.Z. Zubialevich, H. Li, N. Petkov, J.D. Holmes, P.J. Parbrook, Epitaxial lateral overgrowth of AlN on self-assembled patterned nanorods, Journal of Materials Chemistry C, 3 (2016) 431-437.

[24] M. Conroy, V.Z. Zubialevich, H. Li, N. Petkov, S. O'Donoghue, J.D. Holmes, P.J. Parbrook, Ultra-High-Density Arrays of Defect-Free AlN Nanorods: A "Space-Filling" Approach, ACS Nano, 10 (2016) 1988-1994.

[25] V.Z. Zubialevich, P. Pampili, M. McLaren, M. Arredondo-Arechavala, G. Sabui, Z. Shen, P.J. Parbrook, Dense GaN nanocolumn arrays by hybrid top-down-regrow approach using nanosphere lithography, in: 2018 IEEE 18th International Conference on Nanotechnology (IEEE-NANO), IEEE, 2018.

[26] V.Z. Zubialevich, P. Pampili, M. Akhter, I. Izadi, M. McLaren, M.A. Arechavala, P.J. Parbrook, Dense arrays of GaN nanocolumns fabricated by a hybrid top-downregrowth method, In preparation, (2020). 
[27] V.Z. Zubialevich, P. Pampili, P.J. Parbrook, Thermal Stability of Crystallographic Planes of GaN Nanocolumns and Their Lateral Overgrowth by MOVPE, Submitted to Crystal Growth \& Design, (2019).

[28] U. Martin, U. Mühle, H. Oettel, The Quantitative Measurement of Dislocation Density in the Transmission Electron Microscope, Practical metallography, (1995).

[29] A. Pandey, B.S. Yadav, D.V.S. Rao, D. Kaur, A.K. Kapoor, Dislocation density investigation on MOCVD-grown GaN epitaxial layers using wet and dry defect selective etching, Applied Physics A, 122 (2016) 614.

[30] A. Dadgar, P. Veit, F. Schulze, J. Bläsing, A. Krtschil, H. Witte, A. Diez, T. Hempel, J. Christen, R. Clos, A. Krost, MOVPE growth of GaN on $\mathrm{Si}$ - Substrates and strain, Thin Solid Films, 515 (2007) 4356-4361.

[31] K. Forghani, L. Schade, U.T. Schwarz, F. Lipski, O. Klein, U. Kaiser, F. Scholz, Strain and defects in Si-doped (Al)GaN epitaxial layers, Journal of Applied Physics, 112 (2012) 093102.

[32] C.G.V.d. Walle, J. Neugebauer, First-principles calculations for defects and impurities: Applications to III-nitrides, Journal of Applied Physics, 95 (2004) 38513879.

[33] P. Visconti, M.A. Reshchikov, F. Yun, K.M. Jones, H. Morkoç, A. Passaseo, E. Piscopiello, A. Pomarico, R. Cingolani, M. Lomascolo, M. Catalano, Structural, optical and electrical properties of GaN films grown by metalorganic chemical vapor deposition on sapphire, MRS Proceedings, 680 (2011) E3.8.

[34] Q. Li, J.B. Wright, W.W. Chow, T.S. Luk, I. Brener, L.F. Lester, G.T. Wang, Singlemode GaN nanowire lasers, Opt. Express, 20 (2012) 17873-17879.

[35] V. Darakchieva, B. Monemar, A. Usui, On the lattice parameters of GaN, Applied Physics Letters, 91 (2007) 031911.

[36] D. Nilsson, E. Janzén, A. Kakanakova-Georgieva, Lattice parameters of AlN bulk, homoepitaxial and heteroepitaxial material, Journal of Physics D: Applied Physics, 49 (2016) 175108.

[37] H. Shin, K. Jeon, Y. Jang, M. Gang, M. Choi, W. Park, K. Park, Comparison of the microstructural characterizations of GaN layers grown on Si (111) and on sapphire, Journal of the Korean Physical Society, 63 (2013) 1621-1624.

[38] M.A. Moram, M.J. Kappers, F. Massabuau, R.A. Oliver, C.J. Humphreys, The effects of Si doping on dislocation movement and tensile stress in GaN films, Journal of Applied Physics, 109 (2011) 073509.

[39] J. Elsner, R. Jones, P.K. Sitch, V.D. Porezag, M. Elstner, T. Frauenheim, M.I. Heggie, S. Öberg, P.R. Briddon, Theory of Threading Edge and Screw Dislocations in GaN, Physical Review Letters, 79 (1997) 3672-3675.

[40] G. Schoeck, The Peierls dislocation: Line energy, line tension, dissociation and deviation, Acta Materialia, 45 (1997) 2597-2605.

[41] Z. Chen, L.W. Su, J.Y. Shi, X.L. Wang, C.L. Tang, P. Gao, AFM Application in IIINitride Materials and Devices, in: V. Bellitto (Ed.) Atomic Force Microscopy, IntechOpen, Rijeka, 2012, pp. 189-208.

[42] A. Roshko, M. Brubaker, P. Blanchard, T. Harvey, K.A. Bertness, Selective Area Growth and Structural Characterization of GaN Nanostructures on Si(111) Substrates, Crystals, 8 (2018) 366.

[43] W. Ye, A. Ougazzaden, M. Cherkaoui, Analytical formulations of image forces on dislocations with surface stress in nanowires and nanorods, International Journal of Solids and Structures, 50 (2013) 4341-4348. 
[44] P. Khanikar, A. Kumar, A. Subramaniam, Image forces on edge dislocations: a revisit of the fundamental concept with special regard to nanocrystals, Philosophical Magazine, 91 (2011) 730-750.

[45] G. Sabui, V.Z. Zubialevich, M. White, P. Pampili, P.J. Parbrook, M. McLaren, M. Arredondo-Arechavala, Z.J. Shen, GaN Nanowire Schottky Barrier Diodes, IEEE Transactions on Electron Devices, 64 (2017) 2283-2290. 


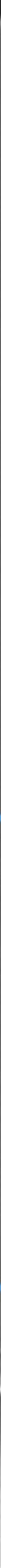




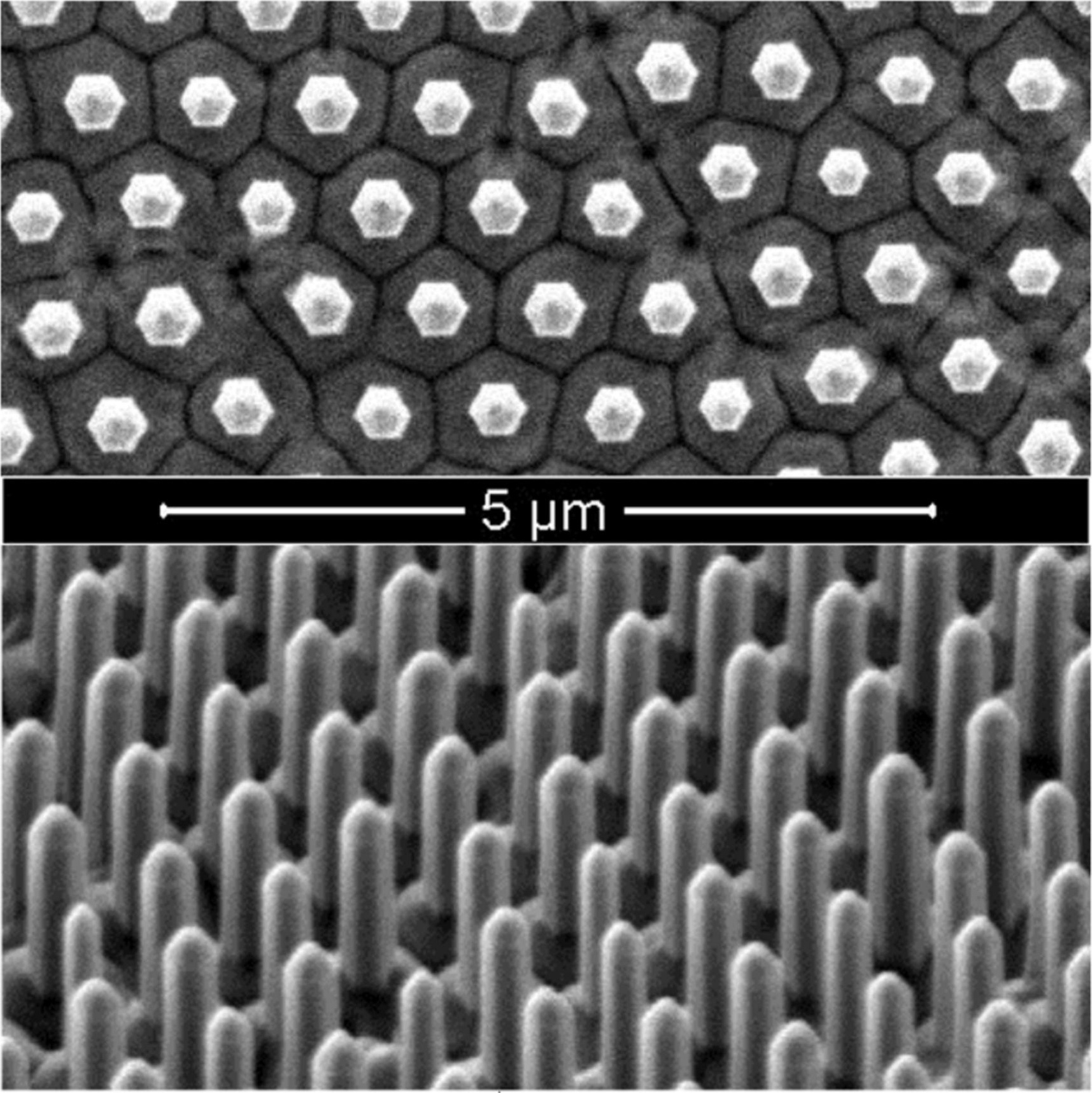




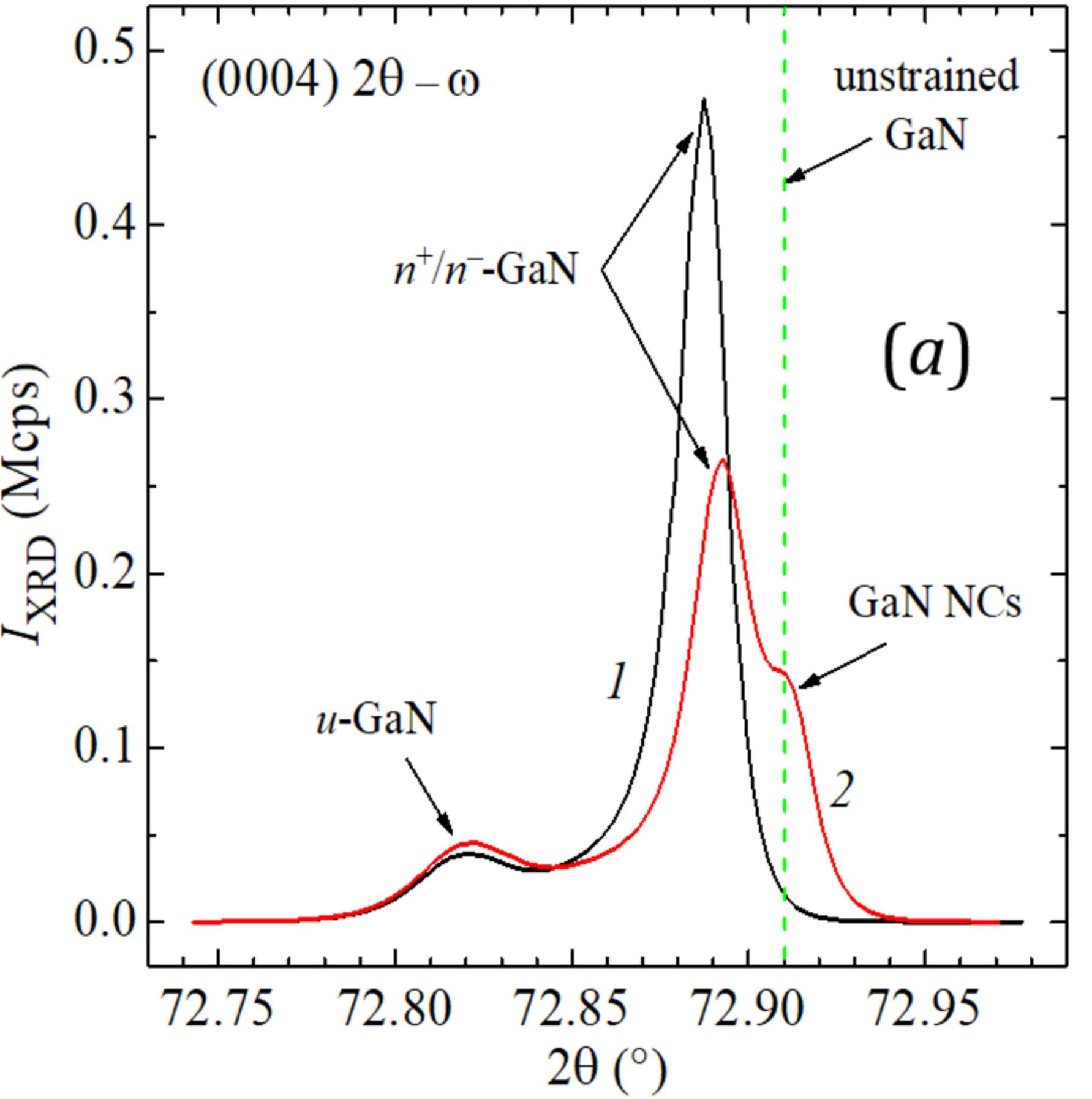

Qy¹0000(rlu)

(b)

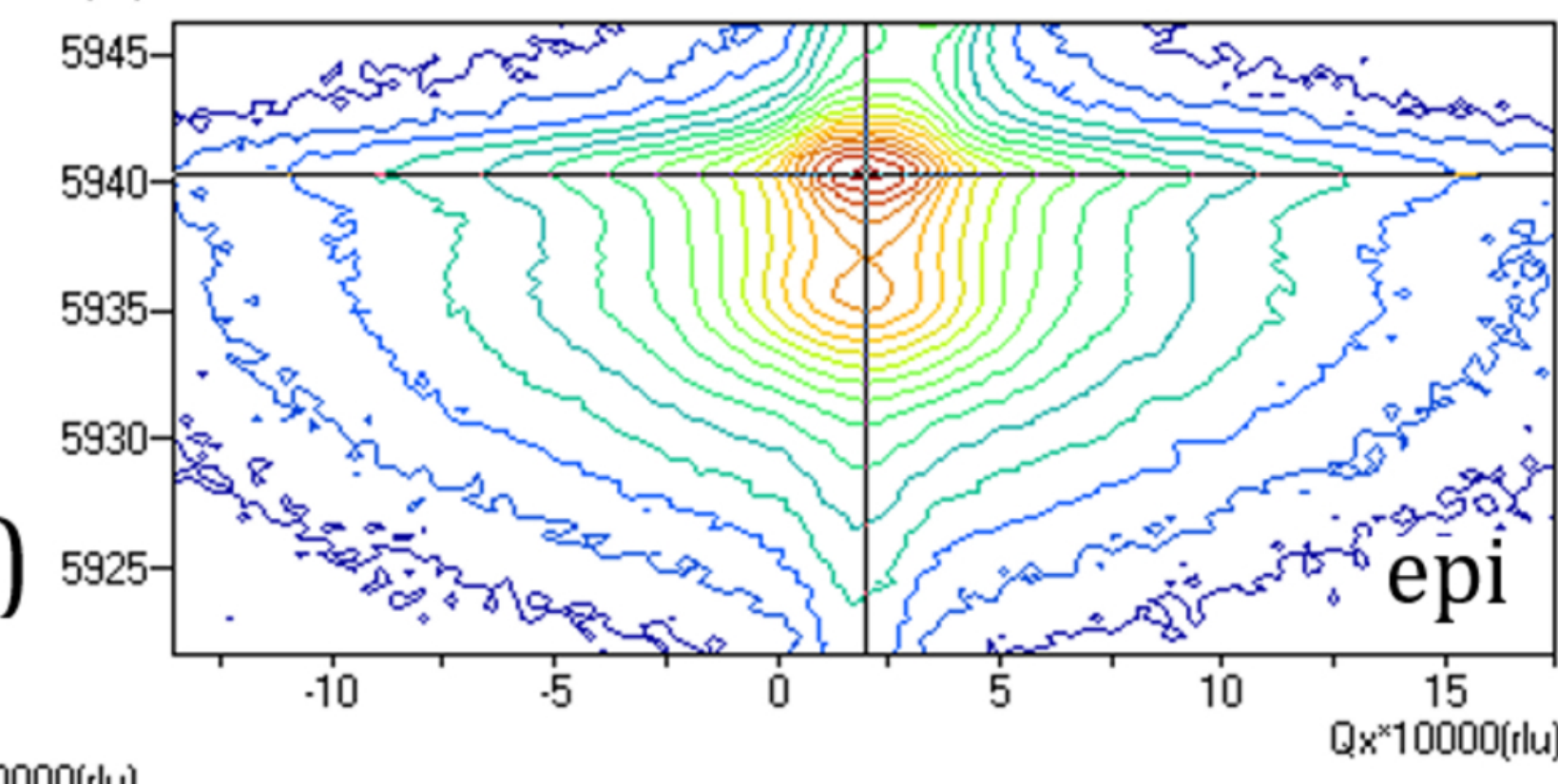

Q $y^{\times 10000(r l u] ~}$

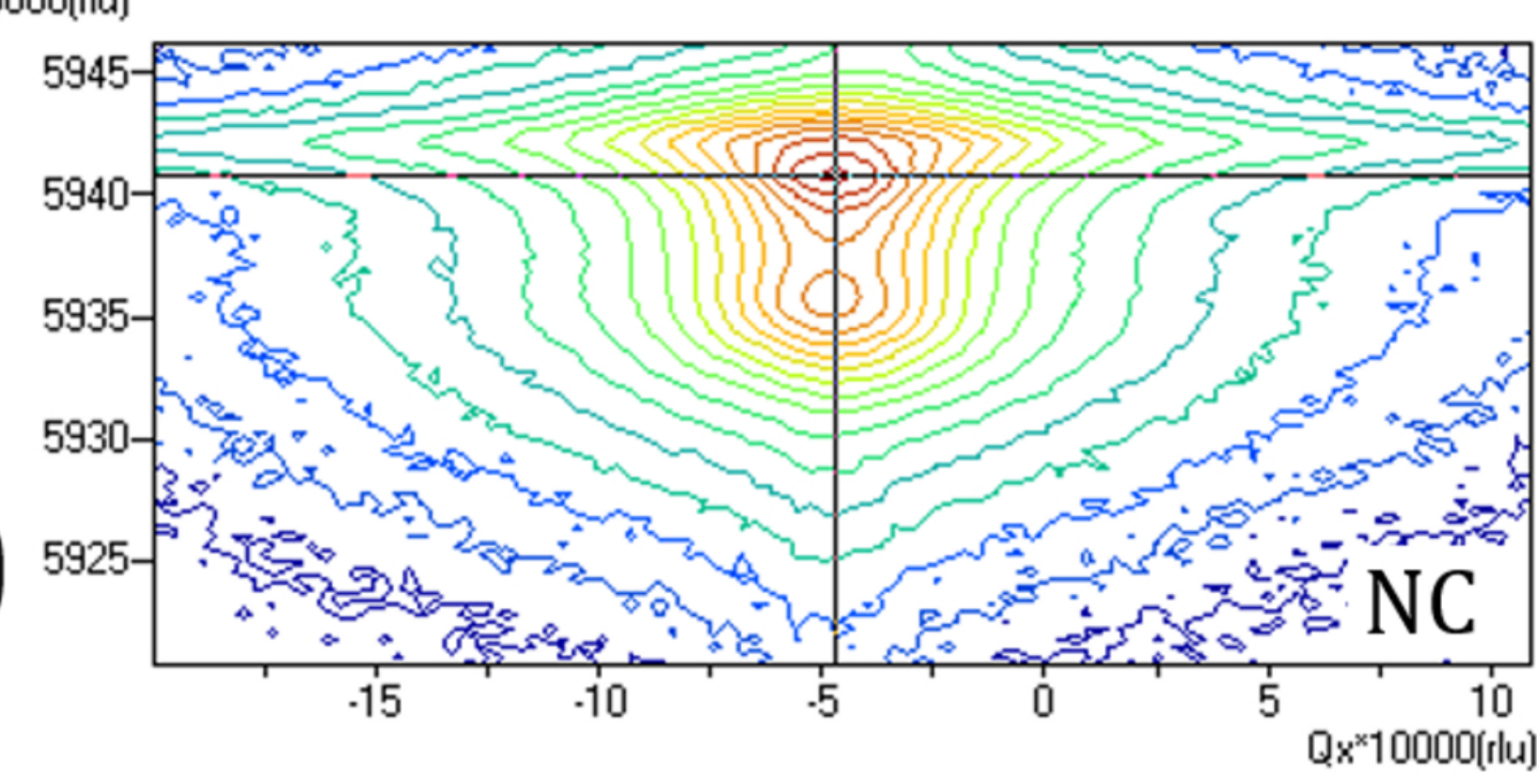

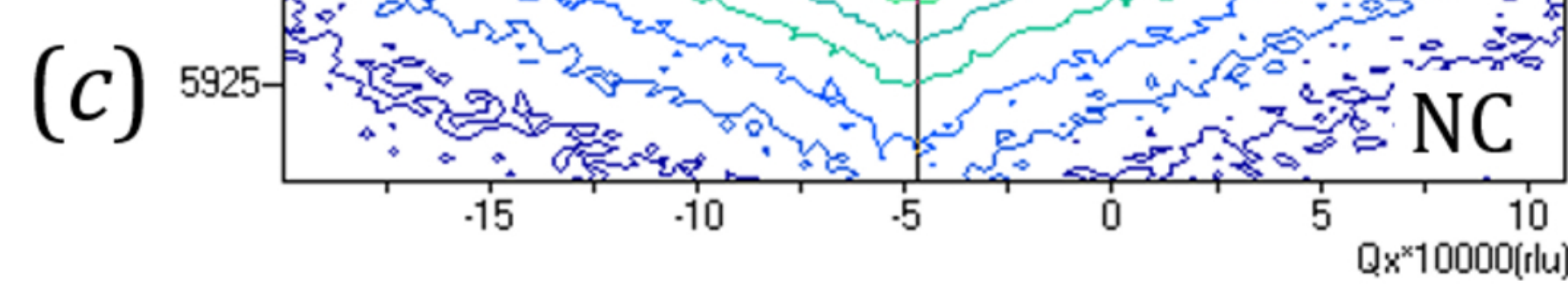





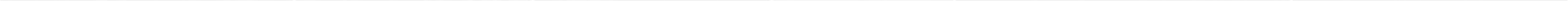

